\title{
A Comprehensive Analysis of Protein Data Bank reveals Low Desolvation Penalty in $\pi$-Cation System
}

\section{Jing-Fang Yang}

Central China Normal University https://orcid.org/0000-0002-3507-1664

\section{Meng-Yao Wang}

Central China Normal University https://orcid.org/0000-0003-2664-9214

\section{Di Wang}

Central China Normal University https://orcid.org/0000-0003-0870-3934

Jing-Yi Li

Central China Normal University https://orcid.org/0000-0002-5672-5209

Ge-Fei Hao ( $\nabla$ gefei_hao@foxmail.com )

Central China Normal University

\section{Qing X. Li}

University of Hawai'i at Manoa Libraries https://orcid.org/0000-0003-4589-2869

\section{Guang-Fu Yang}

Huazhong University of Science and Technology https://orcid.org/0000-0003-4384-2593

\section{Research article}

Keywords: desolvation penalty, $\pi$-cation, cation- $\pi$, molecular interaction, drug and pesticide design

Posted Date: August 28th, 2020

DOI: https://doi.org/10.21203/rs.3.rs-62381/v1

License: () (1) This work is licensed under a Creative Commons Attribution 4.0 International License.

Read Full License 


\section{A comprehensive analysis of Protein Data Bank reveals low desolvation 2 penalty in $\pi$-cation system}

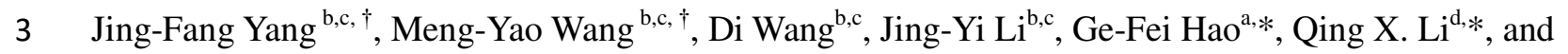

4 Guang-Fu Yang ${ }^{\text {b,c,e }}$

$5 \quad{ }^{a}$ State Key Laboratory Breeding Base of Green Pesticide and Agricultural Bioengineering, Key Laboratory of

6 Green Pesticide and Agricultural Bioengineering, Ministry of Education, Research and Development Center

7 for Fine Chemicals, Guizhou University, Guiyang 550025, P. R. China; ${ }^{b}$ Key Laboratory of Pesticide \&

8 Chemical Biology, Ministry of Education, College of Chemistry, Central China Normal University, Wuhan

9 430079, P.R.China; ${ }^{c}$ International Joint Research Center for Intelligent Biosensor Technology and Health,

10 Central China Normal University, Wuhan, 430079, China; ${ }^{d}$ Department of Molecular Biosciences and

11 Bioengineering, University of Hawaii at Manoa, Honolulu, Hawaii 96822, United States; ${ }^{e}$ Collaborative

12 Innovation Center of Chemical Science and Engineering, Tianjin 300072, P.R.China

13

14

15

16

17

18

19

20

21

22

23

$24{ }^{\dagger}$ Co-first authors.

25 *To whom correspondence should be addressed. E-mail: gefei_hao@foxmail.com;

26 qingl@hawaii.edu

27 
29 Cation- $\pi$ interactions widely exist between ligand-protein interfaces, attracting much attention in 30 molecular recognition in recent years. Interactions named cation- $\pi$ and $\pi$-cation (cationic vs 31 arene small molecular ligands) shall be separately considered in drug and pesticide design 32 process. The two interactions involved in ligands and protein pockets may differ in energy 33 features and therefore offers significant inspiration for drug and pesticide design. However, an

34 in-depth study on differences between cation- $\pi$ and $\pi$-cation systems from an energy perspective 35 is still lacking. In this study, we calculated and compared cation- $\pi$ and $\pi$-cation systems in terms of physicochemical properties of ligand/protein and solvation effect. It seems that the desolvation

37 penalty of the cation- $\pi$ systems was relatively higher than the $\pi$-cation pairs, even though these interactions both can improve the ligand activity. This is the reason for evolution converged on $\pi$-cation interactions in the cation- $\pi$-mediated proteins. The $\pi$-cation interaction facilitating the inhalation of ligand to the pocket may provide a new sight for the molecular design of

41 pharmaceuticals and pesticides.

43 Key Words: desolvation penalty; $\pi$-cation; cation- $\pi$; molecular interaction; drug and pesticide 44 design 


\section{Introduction}

The cation- $\pi$ interaction, dominated by the electrostatic attraction between electron-rich aromatic rings and positively charged groups, is an essential topic in structure-based drug and pesticide design [1-6]. Some instances have been reported in the modulation of receptor and ligand interactions. Douglas et al. found that cation- $\pi$ interactions can modulate the N-methyl-d-aspartate (NMDA) receptor inhibitory potencies of inhaled drugs of abuse [7]. Borissow et al. illustrated that the cation- $\pi$ interaction between adenophostin and Arg504 of Ins $(1,4,5) \mathrm{P} 3 \mathrm{R}$ receptor is responsible for the high potency [8]. Zhu et al. revealed that the cation- $\pi$ interaction between carboxamide fungicides and C_R46 in the Q-site of succinate-ubiquinone oxidoreductase contributes significantly to affinity [9]. Therefore, the cation- $\pi$ interaction plays an important role in modulating molecular recognition between protein and ligand.

The definition of the interactions between cation and $\pi$ in biological systems has experienced a long and complex process. Based on the initial definition from Dougherty et al., cation- $\pi$ interactions can arise between ligands and tryptophan (Trp), tyrosine (Tyr), phenylalanine (Phe), arginine (Arg), and lysine (Lys) [6, 10-12]. Being either cation or $\pi$ ligand can classify such interactions into cation- $\pi$ and $\pi$-cation pairs in drug or pesticide and target interactions (Fig. 1) [13]. The $\pi$ element in a cation- $\pi$ pair is typically provided by the side-chains of aromatic residues, Trp, Tyr, and Phe, while the positively charged ligands act as "cation". For $\pi$-cation pairs, two protonated amino acids (Arg and Lys) are "cation", with arene ligands providing the $\pi$ element [14]. 
71 Recent attention has been paid to study various weak interactions in computational based drug

72 and pesticide design [15-17]. For example, Wendler et al. put forward fitting functions by using

73 different parameters to evaluate energies and detect hydrogen bonds [18]. The fitting functions

74 describe hydrogen bonds in amino acid dimers and the cooperativity of hydrogen bond energies

75 in water clusters quite well [18]. Chourasia et al. constructed an Aromatic-Aromatic Interactions

76 Database and analyzed the connectivity patterns of $\pi-\pi$ networks in proteins [19]. A cluster

77 arrangement of aromatic residues represents a stronger propensity than a linear arrangement. Du

78 et al. proposed an empirical formulation for the interaction energy calculation of cation- $\pi$ in

79 proteins, which can quantitatively determine cation- $\pi$ interactions [20]. The computational

80 approach can be precise in drug and pesticide design. However, the energetic difference between

81 cation- $\pi$ and $\pi$-cation in drug and protein interaction systems remains unclear. While

82 understanding the difference between the two systems makes it easier to use cation- $\pi$ and

$83 \pi$-cation systems for rational drug and pesticide design. Therefore, there is a strong need to

84 perform a systematic analysis of the energetic difference between these two interactions in all

85 available protein structures.

87 In this study, we get 1334 cation- $\pi$ systems and $2174 \pi$-cation systems from 141,706 crystal structures in the PDB database by using Protein-Ligand Interaction Profiler (PLIP) [21]. The

89 physicochemical properties and desolvation penalty were then calculated. Firstly, the physicochemical properties, such as hydrophobicity and the negative decadic logarithm of the

91 ionization constant ( $\mathrm{pKa}$ ) of proteins and ligands, were studied [22-24]. Then, the binding energy

92 was compared. The results showed that $\pi$-cation pairing can sharply reduce the energy of the 93 desolvation penalty, which can facilitate the inhalation of the drug or pesticide into the pocket 
94 and promote the coevolution of proteins and ligands. The reduced desolvation penalty may be

95 induced by the physiochemical property difference between $\pi$-supplier and cation-supplier.

96 Therefore, taking a proper $\pi$-supplier of the ligand scaffold into account will guide how to design

97 potent drugs and pesticides.

Methods

The definition of cation- $\pi$ and $\pi$-cation interactions

The PLIP was used to geometrically identify cation- $\pi$ interactions with default parameters [21]. Furthermore, the cation- $\pi$ and $\pi$-cation interactions were classified by the ligand groups.

103 Tertamine, quartamine, guanidine, and sulfonium ligands provided cations for cation- $\pi$ interactions. Aromatic ligands were $\pi$ components in $\pi$-cation interactions.

\section{The physicochemical property evaluation}

The hydrophobicity of ligand binding pockets was obtained with Fpocket [25]. The pKa was calculated using Hammett-Taft equation derived from plenty of libraries of experimental data. The graphs of descriptors of proteins and ligands related to cation- $\pi$ and $\pi$-cation interactions were analyzed and edited by Origin2019b [26, 27].

\section{Structural optimization}

113 The Sander module of Amber16 was used to minimize the energy of complex structures with 114 implicit solvent [28]. The AMBER ff14SB force field was used for residues, and the general 
115 AMBER force field (gaff ) was used for ligands [29, 30]. The cutoff distance for the long-range 116 electrostatic interaction was set at $10.0 \AA$. The minimization procedure consisted of the following

117 three steps [31]. First, only hydrogens, ions, and water molecules were allowed to move, and the 118 solute remained fixed with a constraint of $500 \mathrm{kcal} \mathrm{mol}^{-1} \AA^{-2}$. Then, the backbone atoms of the 119 protein were fixed, and other atoms were relaxed. Finally, all the atoms of the system were free 120 to move. In each step, the steepest descent method was used for the first 2000 cycles and the 121 conjugate gradient method was used for the next 1000 cycles to perform energy minimization $122[31]$.

\section{Desolvation penalty evaluation}

125 The desolvation penalty (SOL_value) analysis was performed based on Molecular Mechanics 126 Poisson-Boltzmann Surface Area (MM/PBSA), a common method for binding free energy 127 calculation, which is performed using Amber16 software [32].

128 The protein-ligand complex binding energy $\left(\Delta G_{\text {bind }}\right)$ was estimated by the molecular mechanical 129 (MM) gas-phase binding energy $\left(\Delta E_{\mathrm{MM}}\right)$ and solvation energy (desolvation penalty) $\left(\Delta G_{\text {solv }}\right)$ 130 shown in Equation 1 [27].

$131 \Delta G_{\text {bind }}=\Delta E_{\mathrm{MM}}+\Delta G_{\text {solv }}$

132 The $\Delta E_{\mathrm{MM}}$ can be evaluated as the sum of the electrostatic energy $\left(\Delta E_{\text {ele }}\right)$, van der Waals

133 interaction energy $\left(\Delta E_{\mathrm{vdw}}\right)$ and the bond, angle, dihedral energies $\left(\Delta E_{\text {int }}\right)$ according to Equation 2. 134 The $\Delta G_{\text {solv }}$ can be divided into two parts - the electrostatic desolvation penalty $\left(\Delta G_{\mathrm{PB} / \mathrm{GB}}\right)$ and the 135 nonpolar desolvation penalty $\left(\Delta G_{\mathrm{np}}\right)$ (Equation 3). 

ligand and receptor interactions.

\section{Results and discussion}

\section{Data content and analysis}

$\Delta G_{\mathrm{solv}}=\Delta G_{\mathrm{PB} / \mathrm{GB}}+\Delta G_{\mathrm{np}}$

$\Delta E_{\mathrm{MM}}=\Delta E_{\mathrm{int}}+\Delta E_{\mathrm{ele}}+\Delta E_{\mathrm{vdw}}$

The SOL_value was defined as a partition ratio of the solvation energy $\left(\Delta G_{\text {solv }}\right)$ in the sum of the molecular mechanical gas-phase binding energy $\left(\Delta E_{\mathrm{MM}}\right)$ and the solvation energy $\left(\Delta G_{\mathrm{solv}}\right)$ by using Equation 4.

$\mathrm{SOL} \_$value $=\left(\left|\Delta G_{\text {solv }}\right|\right) /\left(\left|\Delta E_{\mathrm{MM}}\right|+\left|\Delta G_{\text {solv }}\right|\right)$

To identify the difference between cation- $\pi$ and $\pi$-cation interactions, we developed a workflow to collect cation- $\pi$ and $\pi$-cation complex crystal structures from Protein Data Bank (PDB) (Fig. 2a). Approximately $60000 \mathrm{pdb}$ files with small molecules were filtered out from 141706 crystal structures downloaded from the PDB database. Then, 8263 complex crystal structures with cation- $\pi$ and $\pi$-cation interactions were geometrically identified through the PLIP. Finally, 1334 complexes with 1926 receptor-ligand cation- $\pi$ interactions and 2174 complexes involved in 2643 receptor-ligand $\pi$-cation interactions were collected and classified according to the ligand groups. The number of complex crystal structures with $\pi$-cation interactions was about 1.63 times of that with cation- $\pi$, indicating that the $\pi$-cation interaction was the more important component in the 
157 of Phe $(324,16.82 \%)$ and Tyr $(327,16.98 \%)$ was absolutely lower than that of $\operatorname{Trp}(1275$,

$15866.20 \%$ ) in cation- $\pi$ interactions. For the $\pi$-cation interactions, the percentage of Arg was about

$15967.42 \%$, which was relatively higher than that of Lys (32.58\%, Fig. 2b). These results implicated

160 that Trp and Arg were essential in the cation- $\pi$ and $\pi$-cation interactions, which was consistent

161 with the previous results [33-35].

162

163 Properties of protein pockets and ligands

164 As we all known, the chemistry community recognized the cation- $\pi$ interaction as a major force

165 for molecular recognition, joining the hydrophobic effect [36]. Therefore, the hydrophobicity, 166 hydrophily, and other properties of proteins and ligands were evaluated.

168 The hydrophobicity score of ligand binding pockets was calculated based on the method 169 proposed by Monera et al $[22,37]$. The higher score represents higher hydrophobicity. The 170 calculated hydrophobic scores of two systems both ranged from -15 to 65 with the normal 171 distribution (Fig. 3a). However, there was a slight difference between cation- $\pi$ and $\pi$-cation: the 172 hydrophobic scores of $\pi$-cation pairs clustered in the interval of -5 to 45 , while the most scores of 173 cation- $\pi$ moved to the interval of 5 to 45 . From violin plots, we found that most score (cation- $\pi$ : $17479.60 \%, \pi$-cation: $77.60 \%$ ) lied in the range of 5-35 (Fig. 3b). Nevertheless, the shape of $\pi$-cation 175 moved down compared with that of cation- $\pi$, revealing that the binding pockets of cation- $\pi$ were 176 much more hydrophobic. 
178 The hydrogen ion is the common proton in the ligands. The $\mathrm{pKa}$ is always used to describe the 179 ability of acid dissociation, which is related to the solubility of the ligand [24, 38]. The smaller

180 the value of $\mathrm{pKa}$ indicates the stronger the acid, in turn, the stronger base[39]. Hence, the pKa of 181 ligands was studied to make the difference between cation- $\pi$ and $\pi$-cation systems. Just like the 182 hydrophobic score, the ligands in $\pi$-cation pairs were the lower pKa biased (Fig. 4a). When the $183 \mathrm{pKa}$ was in the range 0 to 25 , the possession percentage of $\pi$-cation systems was lower than the 184 cation- $\pi$. For the range -15 to 0 , the possession percentage of $\pi$-cation systems was much larger 185 than that of cation- $\pi$. Fig. $4 \mathrm{~b}$ showed the different shapes of two violin plots. In the $\pi$-cation 186 system, there were two wide shapes in the interval of -10 to 0 and the interval of 0 to 10 . For the cation- $\pi$ systems, three wider sections were shown. Meanwhile, the first wide part of cation- $\pi$ was relatively higher than that of $\pi$-cation. On the other hand, the last wide shape of $\pi$-cation was lower than the cation- $\pi$. The median of cation- $\pi(\sim 10)$ was higher than $\pi$-cation $(\sim 5)$. These results indicated that the basic ligands preferred to act as cations in cation- $\pi$ systems.

\section{Desolvation penalty difference}

Solvation energy is a fundamental thermodynamic quantity to estimate the desolvation cost of a 194 ligand-binding with a protein [40]. The effect of the desolvation penalty is important in drug discovery due to its influence on the inhalation of a drug into the pocket [41]. Accounting for the 196 effect of solvent on the strength of molecular interactions has been a long-standing problem for 197 structure-based drug design. From the above, we found that the strong basic ligands preferred to 198 bind with the hydrophobic binding pockets to form cation- $\pi$, vice versa, the $\pi$-cation interaction would be formed. Hence, the solvent may be a dominant force to dictate the interaction of molecules and proteins with the opposite solubilities. Actually, the solvent exposure phenomenon 
201 is frequently found in the residues involved in cation- $\pi$ or $\pi$-cation interactions, and the

202 surrounding solvents may be one of the main factors to modulate the strength of cation- $\pi$ or

$203 \pi$-cation interactions $[42,43]$. In particular, some theoretical studies have established the

204 importance of the desolvation penalty for cation- $\pi$ systems [42, 44-47]. However, whether there

205 is a difference in the desolvation penalty between cation- $\pi$ and $\pi$-cation systems is still unknown.

206

207 In this study, the SOL_value, avoiding the system interference, was defined to determine the

208 overall influence of the desolvation penalty for the binding affinity according to Equation 4 . If

209 the SOL_value is close to 0.50 , the absolute value of $\Delta G_{\text {solv }}$ is clearly close to the absolute value

210 of $\Delta E_{\mathrm{MM}}$. In contrast, if the SOL_value tends to 0.00 , the $\Delta G_{\text {solv }}$ is definitely close to 0.00 ,

211 resulting in a lower desolvation penalty for the binding affinity.

212

213 The distributions of SOL_value of 1334 complexes with cation- $\pi$ interactions and 2174

214 complexes with $\pi$-cation interactions were analyzed. As shown in Fig. 5a, the SOL_value ranged

215 from 0.00 to 0.50 with 0.45 as a demarcation point. When the SOL_value was larger than 0.45 ,

216 there was more cation- $\pi$ interaction $(45.50 \%)$ than $\pi$-cation interaction $(27.37 \%)$. When the

217 SOL_value is ranged from 0.45 to 0.47 , the percentage of cation- $\pi(26.76 \%)$ was much larger

218 than that of $\pi$-cation $(13.01 \%)$. In contrast, the percentage of $\pi$-cation was larger than the

219 cation- $\pi$ in the SOL_value ranges of $0.00 \sim 0.30,0.30 \sim 0.40$, and $0.40 \sim 0.45$. The percentages of

$220 \pi$-cation were $25.71 \%, 22.63 \%$, and $24.29 \%$, respectively. The largest difference appears in the

221 SOL_value range of $0.00 \sim 0.30$, in which the percentage of $\pi$-cation was $10.64 \%$ higher than that

222 of cation- $\pi$. The bottom of the violin plot of cation- $\pi$ was much thinner than that of $\pi$-cation

223 systems, which demonstrates that there were fewer values in comparison to $\pi$-cation systems 
224 when tending to 0.00 (Fig. 5b). In summary, the SOL_values of over $72 \%$ of $\pi$-cation systems

225 are much closer to 0.00 . This means that the $\pi$-cation interactions in the complexes may result in 226 a lower desolvation penalty which is conducive to the binding affinity. It is maybe the reason for 227 the enrichment of $\pi$-cation in the natural systems.

\section{Case study}

The above results revealed that the hydrophobicity of cation- $\pi$ pockets was relatively higher, while the strong basic cation ligands in cation- $\pi$ pairs had a stronger ability to dissolve in the water. These properties led to the higher solvent effect in cation- $\pi$ interactions. To further illustrate the values of $\pi$-cation and cation- $\pi$ interactions, we collected a series of representative crystal structures with available experimental binding affinities, such as, the half-maximum inhibition concentration $\left(I C_{50}\right)$ and the inhibitory constant $\left(K_{i}\right)$ (Table $\mathrm{S} 1$ and $\mathrm{S} 2$ ). Further, we used two cases to explain the difference between cation- $\pi$ and $\pi$-cation in the protein systems

237 (Fig. 6a and b). Serine/threonine kinase acts as an essential component of the mitogen-activated protein kinase (MAPK) signal transduction pathway. MAPK1/ERK2 is one of the two MAPKs playing an important role in the MAPK/ERK cascade and is a key oncogenic pathway implicated in a variety of human cancers. The $K_{i}$ of ligand 33A was reduced from $2300 \mathrm{nM}$ to $86 \mathrm{nM}$ after the chlorine and benzene ring substitution [48]. The benzene ring of 33A formed $\pi$-cation interactions with Lys52. On the other hand, the leukotriene 4 hydrolase (LTA4H) is a key target

243 for the treatment of cardiovascular disease. The binding affinity of $27 \mathrm{P}\left(I C_{50}=26 \mathrm{nM}\right)$ was 244 improved after the modification of $24 \mathrm{P}\left(I C_{50}=87 \mathrm{nM}\right)[49]$. The nitrogen atom formed cation- $\pi$ 245 interaction with Tyr267 and Tyr378. It was common that the hydrophobicity score of the 246 MAPK1 pocket for $\pi$-cation pair was lower than that of LTA4H for cation- $\pi$. Meanwhile, the 
247 SOL_value was improved after the introduction of cation- $\pi$ and $\pi$-cation interactions. However, 248 these interactions both can strengthen the interaction of the ligand with receptors, even though

249 the SOL_value of 27P-LTA4H (0.41) was much higher than 33A-MAPK1 (0.18). Meanwhile, it 250 should be noted that the activity fold changed of 33A (26.74) was much higher than 27P (3.35).

251 These results are consistent with our conclusion that the $\pi$-cation interaction has a lower

252 desolvation penalty than cation- $\pi$ systems, which would contribute to the ligand binding.

\section{Conclusion}

255 The cation and $\pi$ interactions play an essential role in drug(pesticide)-target interaction.

256 Differentiating cation- $\pi$ and $\pi$-cation systems can facilitate the rational molecular design of

257 pharmaceuticals and pesticides. We comprehensively compared the physicochemical properties

258 of protein pockets and ligands and solvation energy for cation- $\pi$ and $\pi$-cation systems in this

259 study. Compared with a cation- $\pi$ system, a $\pi$-cation system probably results in a lower

260 desolvation penalty, which may facilitate the inhalation of ligand to the pocket and determine the

261 coevolution of proteins and ligands. Due to the dependence upon the binding site of the target

262 proteins in drug and pesticide design, the $\pi$-cation interaction is a valuable tool when the ligand

263 contains an aromatic functional group. Therefore, taking a proper $\pi$-supplier of the ligand

264 scaffold into account can guide drug and pesticide design.

266 Abbreviations

267 NMDA: N-methyl-d-aspartate; Trp: tryptophan; Tyr: tyrosine; Phe: phenylalanine; Arg: arginine; 268 Lys: lysine; pKa: the ionization constant; MM/PBSA: Molecular Mechanics Poisson-Boltzmann 
269 Surface Area; PDB: Protein Data Bank; $I C_{50}$ : the half-maximum inhibition concentration; $K_{i}$ : the 270 inhibitory constant; MAPK: the mitogen-activated protein kinase; LTA4H: the leukotriene 4 271 hydrolase

272

273 Acknowledgements

274 None.

275

276 Author's contributions

277 All authors have contributed to the manuscript and given approval to the final version of the 278 manuscript.

279

280 Funding

281 This work was supported in part by the National Key R\&D Program (2017YFD0200501) and the 282 National Natural Science Foundation of China (21772059, 91853127, and 31960548). Program

283 of Introducing Talents of Discipline to Universities of China (111 Program, D20023). The USDA 284 (Hatch HAW5032-R), and Hawaii Community Foundation (18ADV-90801).

286 Competing interests

287 The authors declare that they have no known competing financial interests or personal 288 relationships that could have appeared to influence the work reported in this paper. 


\section{References}

290 1. Mecozzi S, West AP, Jr., Dougherty DA (1996) Cation-pi interactions in aromatics of biological and medicinal interest: electrostatic potential surfaces as a useful qualitative guide. Proc Natl Acad Sci U S A

2. Pless SA, Millen KS, Hanek AP et al (2008) A Cation-pi Interaction in the Binding Site of the Glycine Receptor Is Mediated by a Phenylalanine Residue. J Neurosci 28(43):10937-10942.

295

3. Zhou Y, Xi Z, Chen W, Wang D (2008) Dinickel(II) Complexes of Bis(N-heterocyclic carbene) Ligands Containing Ni-2(mu-OH) Cores as Highly Efficient Catalysts for the Coupling of Aryl Chlorides. Organometallics 27(22):5911-5920.

298 4. Dougherty DA (2007) Cation-pi interactions involving aromatic amino acids. J Nutr 137(6):1504S-1508S.

299 5. Schottel BL, Chifotides HT, Dunbar KR (2008) Anion-pi interactions. Chem Soc Rev 37(1):68-83.

300 6. Burley SK, Petsko GA (1986) Amino-aromatic interactions in proteins. FEBS Lett 203(2):139-143.

301 7. Raines DE, Gioia F, Claycomb RJ, Stevens RJ (2004) The N-methyl-D-aspartate receptor inhibitory 302 potencies of aromatic inhaled drugs of abuse: Evidence for modulation by cation-pi interactions. J Pharmacol Exp Ther 311(1):14-21.

8. Borissow CN, Black SJ, Paul M et al (2005) Adenophostin A and analogues modified at the adenine moiety: synthesis, conformational analysis and biological activity. Org Biomol Chem 3(2):245-252.

9. Zhu X-L, Xiong L, Li H et al (2014) Computational and Experimental Insight into the Molecular Mechanism of Carboxamide Inhibitors of Succinate-Ubquinone Oxidoreductase. Chemmedchem 9(7):1512-1521.

309 10. Crowley PB, Golovin A (2005) Cation-pi interactions in protein-protein interfaces. Proteins 59(2):231-239.

310 11. Scrutton NS, Raine AR (1996) Cation-pi bonding and amino-aromatic interactions in the biomolecular recognition of substituted ammonium ligands. Biochem J 319 ( Pt 1):1-8.

312 12. Gallivan JP, Dougherty DA (1999) Cation-pi interactions in structural biology. Proc Natl Acad Sci U S A 96(17):9459-9464.

314 13. Liang Z, Li QX (2018) pi-Cation Interactions in Molecular Recognition: Perspectives on Pharmaceuticals 315 and Pesticides. J Agric Food Chem 66(13):3315-3323.

316 14. Pellequer JL, Zhao B, Kao HI et al (2000) Stabilization of bound polycyclic aromatic hydrocarbons by a 
pi-cation interaction. J Mol Biol 302(3):691-699.

318 15. Meyer EA, Castellano RK, Diederich F (2003) Interactions with aromatic rings in chemical and biological

16. Salonen LM, Ellermann M, Diederich F (2011) Aromatic Rings in Chemical and Biological Recognition: Energetics and Structures. Angew Chem Int Ed 50(21):4808-4842.

322 17. Hao G-F, Jiang W, Ye Y-N et al (2016) ACFIS: a web server for fragment-based drug discovery. Nucleic Acids Res 44(W1):W550-W556.

324 18. Wendler K, Thar J, Zahn S, Kirchner B (2010) Estimating the Hydrogen Bond Energy. J Phys Chem A 114(35):9529-9536.

19. Chourasia M, Sastry GM, Sastry GN (2011) Aromatic-Aromatic Interactions Database, A(2)ID: An analysis of aromatic pi-networks in proteins. Int J Biol Macromol 48(4):540-552.

20. Du Q-S, Long S-Y, Meng J-Z, Huang R-B (2012) Empirical Formulation and Parameterization of Cation-pi Interactions for Protein Modeling. J Comput Chem 33(2):153-162.

21. Salentin S, Schreiber S, Haupt VJ et al (2015) PLIP: fully automated protein-ligand interaction profiler. Nucleic Acids Res 43(W1):W443-W447.

332 22. Monera OD, Sereda TJ, Zhou NE et al (1995) Relationship of sidechain hydrophobicity and alpha-helical propensity on the stability of the single-stranded amphipathic alpha-helix. J Pept Sci 1(5):319-329.

23. Egner U, Hillig RC (2008) A structural biology view of target drugability. Expert Opin Drug Dis

24. Settimo L, Bellman K, Knegtel RMA (2014) Comparison of the Accuracy of Experimental and Predicted pKa Values of Basic and Acidic Compounds. Pharm Res 31(4):1082-1095.

25. Schmidtke P, Le Guilloux V, Maupetit J, Tuffery P (2010) fpocket: online tools for protein ensemble pocket

26. Wang M-y, Wang F, Hao G-F, Yang G-F (2019) FungiPAD: A Free Web Tool for Compound Property Evaluation and Fungicide-Likeness Analysis. J Agric Food Chem 67(7):1823-1830.

342 27. Wu F-X, Wang F, Yang J-F et al (2018) AIMMS suite: a web server dedicated for prediction of drug resistance on protein mutation. Brief Bioinformatics 21(1):318-328.

344 28. Case DA, Cheatham TE, Darden T et al (2005) The Amber biomolecular simulation programs. J Comput 
Chem 26(16):1668-1688.

29. Maier JA, Martinez C, Kasavajhala K et al (2015) ff14SB: Improving the Accuracy of Protein Side Chain and Backbone Parameters from ff99SB. J Chem Theory Comput 11(8):3696-3713.

30. Wang JM, Wolf RM, Caldwell JW et al (2004) Development and testing of a general amber force field. J Comput Chem 25(9):1157-1174.

31. Wang F, Wu F-X, Li C-Z et al (2019) ACID: a free tool for drug repurposing using consensus inverse docking strategy. J Cheminformatics 11(1):73.

32. Genheden S, Ryde U (2015) The MM/PBSA and MM/GBSA methods to estimate ligand-binding affinities. Expert Opin Drug Dis 10(5):449-461.

33. Krone MW, Albanese KI, Leighton GO et al (2020) Thermodynamic consequences of Tyr to Trp mutations in the cation- $\pi$-mediated binding of trimethyllysine by the HP1 chromodomain. Chem Sci 11(13):3495-3500.

34. Li H-L, Ma Y, Ma Y et al (2017) The design of novel inhibitors for treating cancer by targeting CDC25B through disruption of CDC25B-CDK2/Cyclin A interaction using computational approaches. Oncotarget 8(20):33225-33240.

35. Gallivan JP, Dougherty DA (1999) Cation-pi interactions in structural biology. Proc Natl Acad Sci U S A 96(17):9459-9464.

36. Dougherty DA (2013) The cation- $\pi$ interaction. Acc Chem Res 46(4):885-893.

37. Guo Z, Li B, Cheng L-T et al (2015) Identification of Protein-Ligand Binding Sites by the Level-Set Variational Implicit-Solvent Approach. J Chem Theory Comput 11(2):753-765.

38. Wang F, Yang J-F, Wang M-Y et al (2020) Graph attention convolutional neural network model for chemical poisoning of honey bees' prediction. Sci Bull.

39. Bandyopadhyay D, Bhatnagar A, Jain S, Pratyaksh P (2020) Selective Stabilization of Aspartic Acid Protonation State within a Given Protein Conformation Occurs via Specific "Molecular Association". J Phys Chem B 124(26):5350-5361.

40. Choi H, Kang H, Park H (2013) New solvation free energy function comprising intermolecular solvation and intramolecular self-solvation terms. J Cheminformatics 5:8.

41. Lipinski CA, Lombardo F, Dominy BW, Feeney PJ (2012) Experimental and computational approaches to 
estimate solubility and permeability in drug discovery and development settings. Adv Drug Del Rev 64:4-17.

375 42. Mahadevi AS, Sastry GN (2013) Cation-pi Interaction: Its Role and Relevance in Chemistry, Biology, and Material Science. Chem Rev 113(3):2100-2138.

377

43. Berry BW, Elvekrog MM, Tommos C (2007) Environmental modulation of protein cation-pi interactions. J Am Chem Soc 129(17):5308-5309.

44. Larrucea J, Rezabal E, Marino T et al (2010) Ab Initio Study of Microsolvated Al3+-Aromatic Amino Acid Complexes. J Phys Chem B 114(27):9017-9022.

45. Biot C, Buisine E, Rooman M (2003) Free-energy calculations of protein-ligand cation-pi and amino-pi interactions: From vacuum to proteinlike environments. J Am Chem Soc 125(46):13988-13994.

46. $\mathrm{Xu} \mathrm{YC,} \mathrm{Shen} \mathrm{JH,} \mathrm{Zhu} \mathrm{WL} \mathrm{et} \mathrm{al} \mathrm{(2005)} \mathrm{Influence} \mathrm{of} \mathrm{the} \mathrm{water} \mathrm{molecule} \mathrm{on} \mathrm{cation-pi} \mathrm{interaction:} \mathrm{Ab} \mathrm{initio}$

47. Remko M, Soralova S (2012) Effect of water coordination on competition between pi and non-pi cation

48. Aronov AM, Baker C, Bemis GW et al (2007) Flipped Out: Structure-Guided Design of Selective complexes. J Biol Inorg Chem 17(4):621-630. 


\section{Figure legends}

399

400

401

402

403

404

405

406

407

408

409

410

411

412

413

414

415

Fig. 1 Definition of cation- $\pi$ and $\pi$-cation interactions. For the cation- $\pi$ pair, the $\pi$ system is typically provided by the aromatic side-chains of Trp, Tyr, and Phe, while the protonated ligand exists as the cation (PDB code: 1ax9). In the $\pi$-cation system, protonated Arg and Lys always act as cations with the arene ligands acting as a $\pi$ partner (PDB code: $1 \mathrm{bkm})$.

Fig. 2 (a) The workflow of screening cation- $\pi$ and $\pi$-cation systems and (b) the number of residue pairs found in cation- $\pi$ and $\pi$-cation systems. First, $141706 \mathrm{X}$-ray structures were downloaded from the PDB database. Then, approximately $60000 \mathrm{pdb}$ files with small molecules were filtered out. Thirdly, 8263 complex crystal structures containing cation- $\pi$ or $\pi$-cation interactions were screened by PLIP package. Finally, 1334 complexes with 1926 receptor-ligand cation- $\pi$ interactions and 2174 complexes involved in 2643 receptor-ligand $\pi$-cation interactions were collected and classified according to the ligand groups.

Fig. 3 The histogram (a) and violin plot (b) of protein pocket hydrophobicity score in cation- $\pi$ and $\pi$-cation systems.

Fig. 4 The histogram (a) and violin plot (b) of ligand $\mathrm{pK}_{\mathrm{a}}$ in cation- $\pi$ and $\pi$-cation systems.

Fig. 5 The histogram (a) and violin plot (b) of SOL_Value in cation- $\pi$ and $\pi$-cation systems.

Fig. 6 (a) Depicts of cation- $\pi$ and $\pi$-cation interactions and (b) comparison of cation- $\pi$ and $\pi$-cation interactions for $I C_{50} / K_{i}$, hydrophobic score (Hyd score), and SOL_Value. 


\begin{tabular}{|c|c|c|c|}
\hline \multicolumn{2}{|c|}{ Cation - $\mathrm{c}$} \\
\hline Residues & Ligand & \multicolumn{2}{c|}{ Residues - Cation } \\
\hline Ligand \\
\hline
\end{tabular}

$417 \quad$ Fig 1.

a

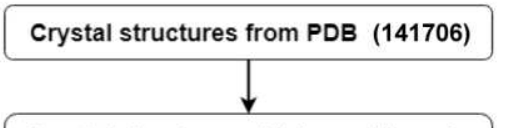

Crystal structures with bound ligands ( 60000)

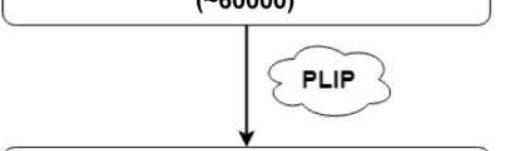

Complex including cation- $\pi$ or $\pi$-cation interactions (8263)

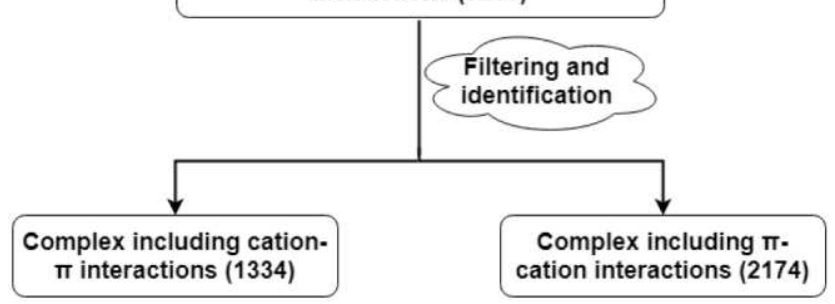

b The number of residue pairs in cation- $\pi$ and $\pi$-cation systems

\begin{tabular}{|c|c|c|c|c|}
\hline \multicolumn{3}{|c|}{ Cation-m (1334) } & \multicolumn{2}{|c|}{$\pi$-cation (2174) } \\
\hline Trp & Tyr & Phe & Arg & Lys \\
\hline 1275 & 327 & 324 & 1782 & 861 \\
\hline
\end{tabular}

\section{$419 \quad$ Fig 2.}



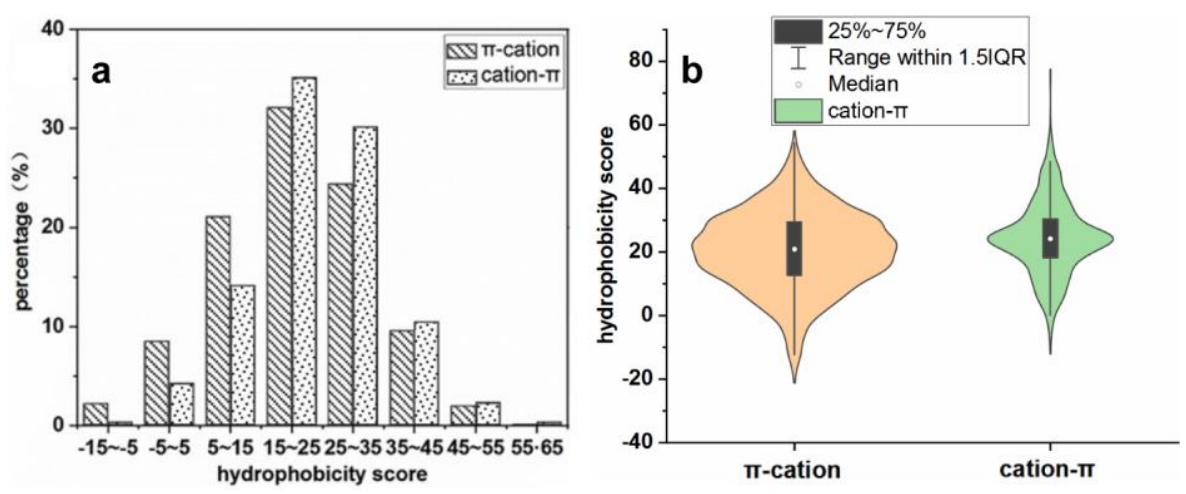

$421 \quad$ Fig 3.

422
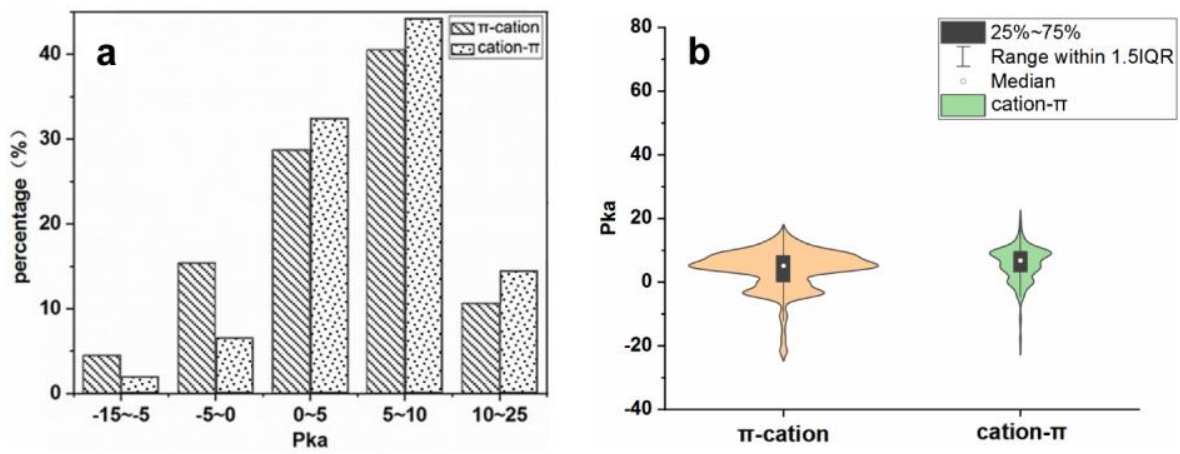

$423 \quad$ Fig 4.
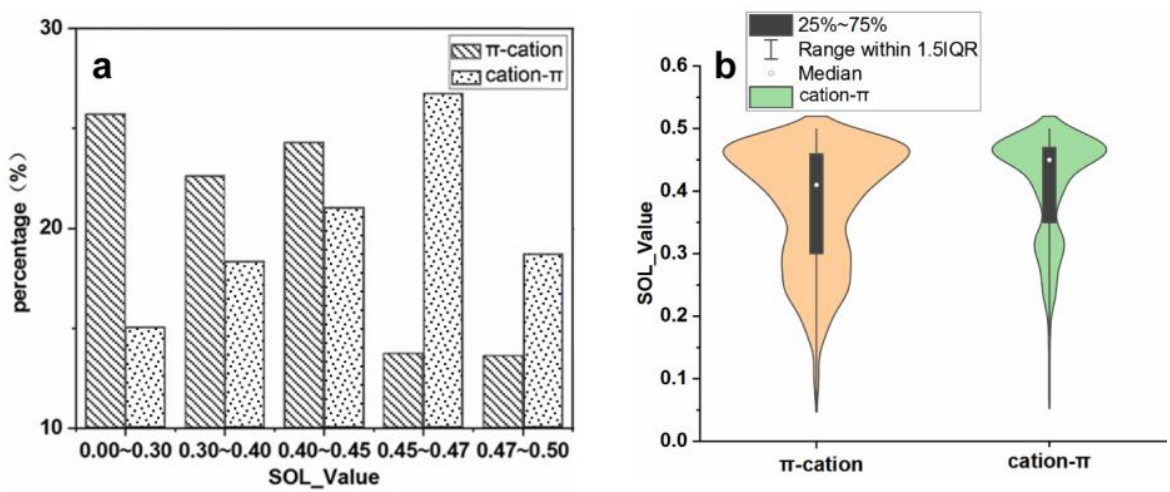

424

$425 \quad$ Fig 5. 
a

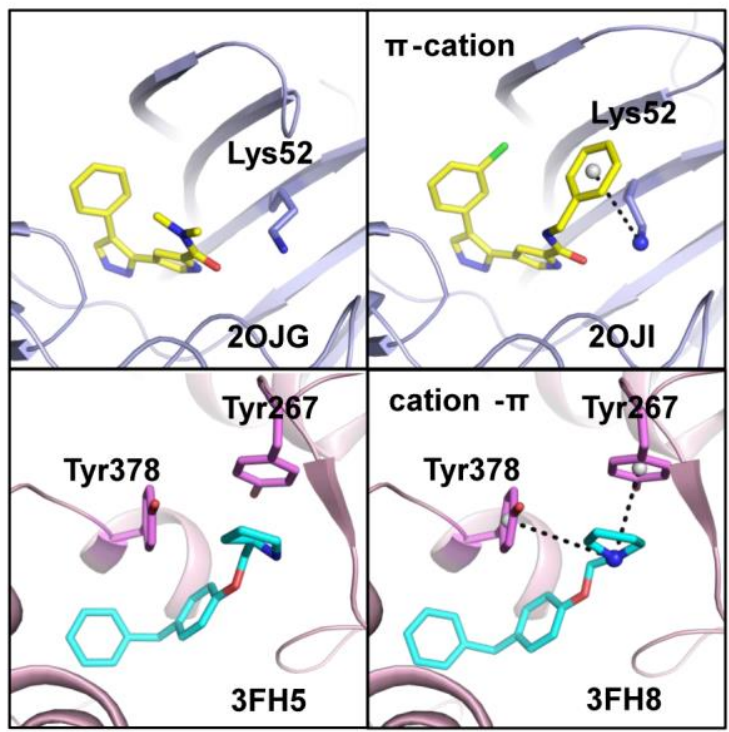

b The comparison of cation $-\pi$ and $\pi$-cation interaction

\begin{tabular}{cccccc}
\hline PDB & $\mathrm{IC}_{50} / K_{i}(\mathrm{nM})$ & Hyd score & SOL_Value & $\pi$ & cation \\
\hline 2OJG & 2300 & 16.39 & 0.15 & & \\
2OJI & 86 & 16.39 & 0.18 & $33 A$ & Lys52 \\
3FH5 & 87 & 42.41 & 0.39 & & \\
3FH8 & 26 & 42.41 & 0.41 & Tyr267/378 & 27P \\
\hline
\end{tabular}

$427 \quad$ Fig 6. 
Figures

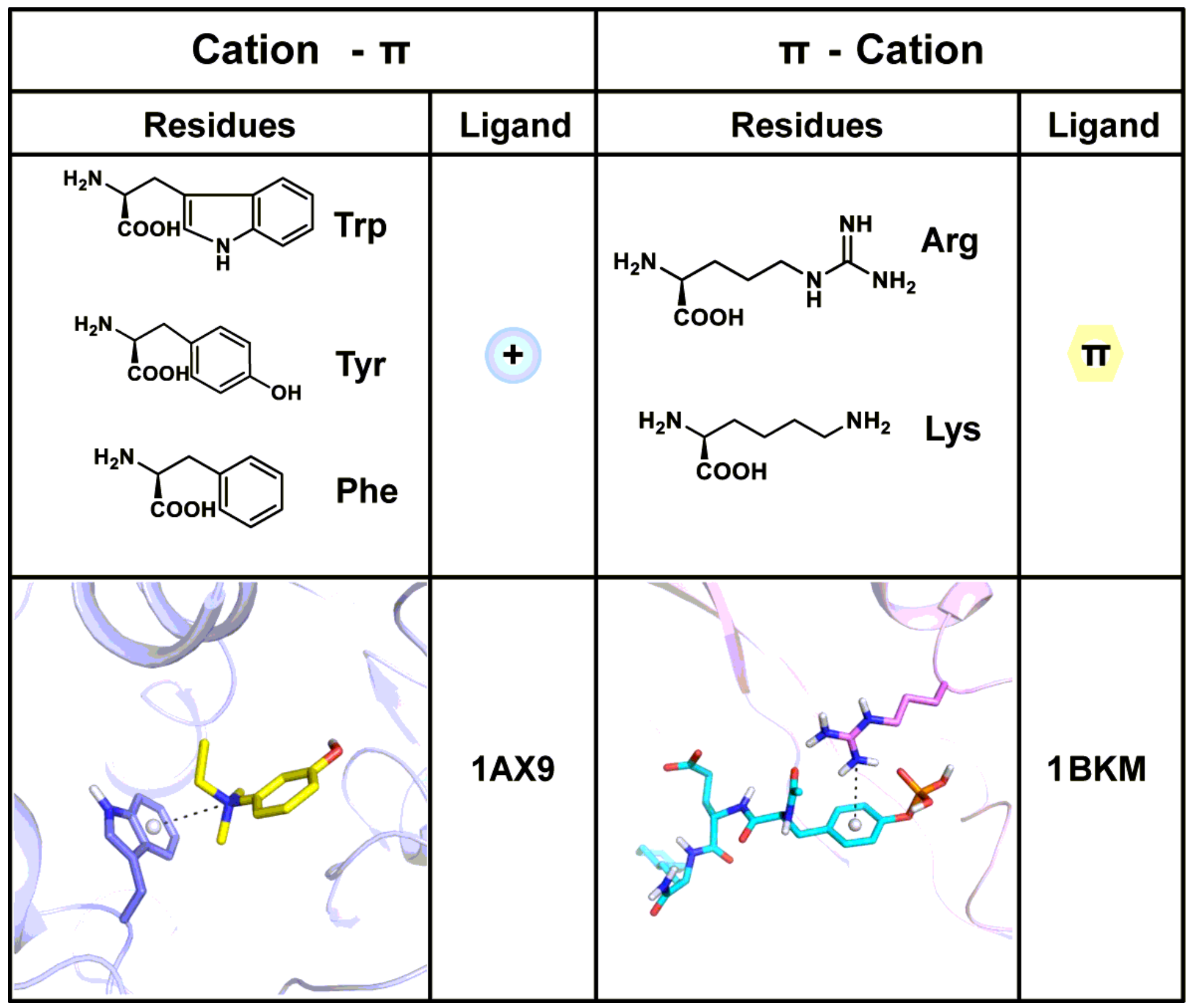

Figure 1

Definition of cation- $\pi$ and $\pi$-cation interactions. For the cation- $\pi$ pair, the $\pi$ system is typically provided by the aromatic side-chains of Trp, Tyr, and Phe, while the protonated ligand exists as the cation (PDB code: 1ax9). In the $\pi$-cation system, protonated Arg and Lys always act as cations with the arene ligands acting as a $\pi$ partner (PDB code: $1 \mathrm{bkm})$. 


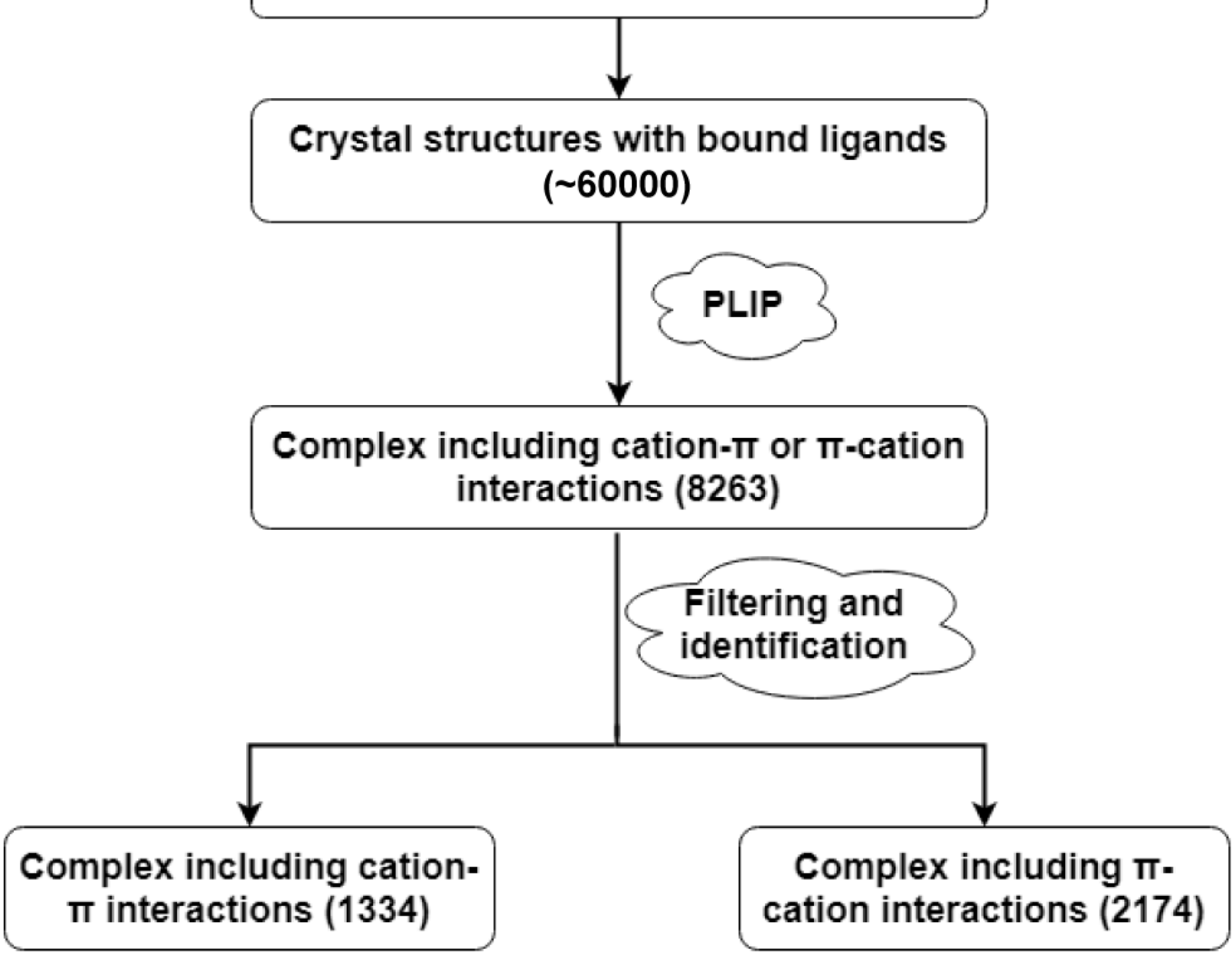

b The number of residue pairs in cation- $\pi$ and $\pi$-cation systems

\begin{tabular}{ccc|cc}
\hline \multicolumn{3}{c|}{ Cation- $\pi$ (1334) } & \multicolumn{2}{c}{$\pi$-cation (2174) } \\
Trp & Tyr & Phe & Arg & Lys \\
\hline 1275 & 327 & 324 & 1782 & 861 \\
\hline
\end{tabular}

Figure 2

(a) The workflow of screening cation- $\pi$ and $\pi$-cation systems and (b) the number of residue pairs found in cation- $\pi$ and $\pi$-cation systems. First, 141706 X-ray structures were downloaded from the PDB database. Then, approximately $60000 \mathrm{pdb}$ files with small molecules were filtered out. Thirdly, 8263 complex crystal structures containing cation- $\pi$ or $\pi$-cation interactions were screened by PLIP package. 
Finally, 1334 complexes with 1926 receptor-ligand cation- $\pi$ interactions and 2174 complexes involved in 2643 receptor-ligand $\pi$-cation interactions were collected and classified according to the ligand groups.
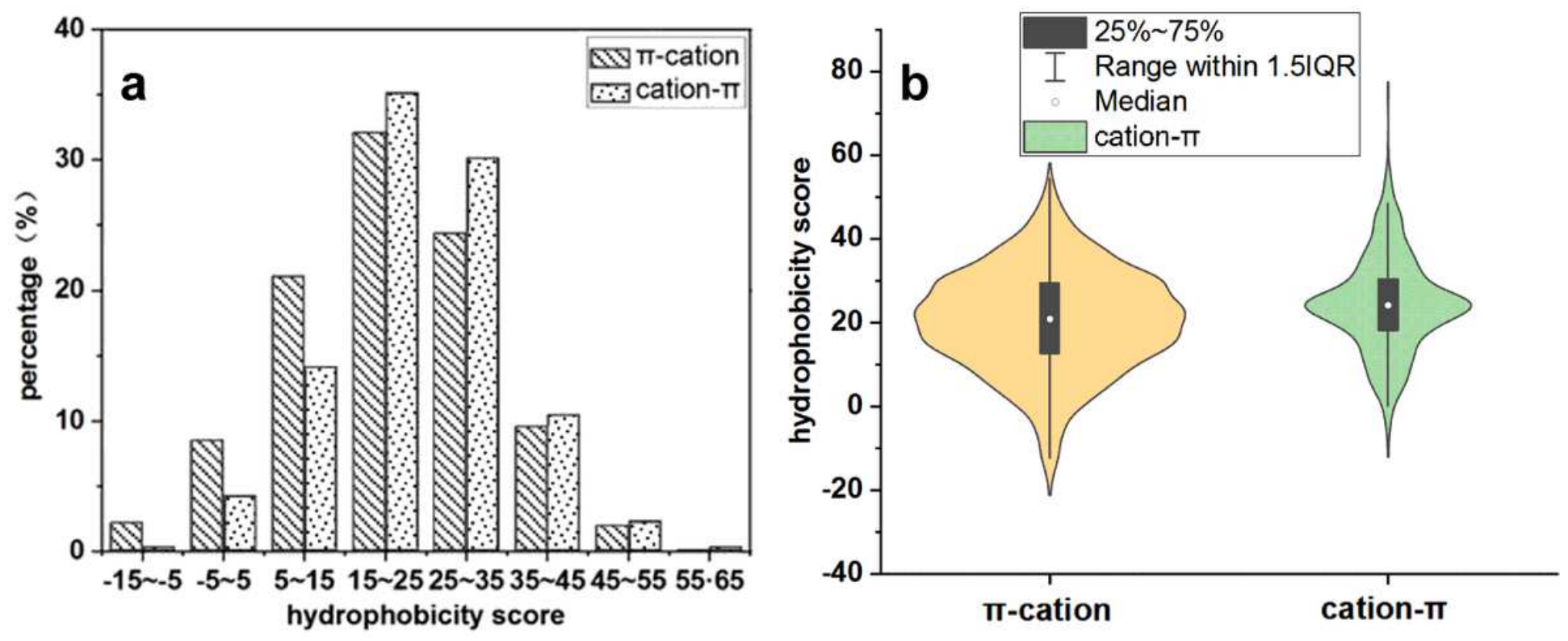

Figure 3

The histogram (a) and violin plot (b) of protein pocket hydrophobicity score in cation- $\pi$ and $\pi$-cation systems.
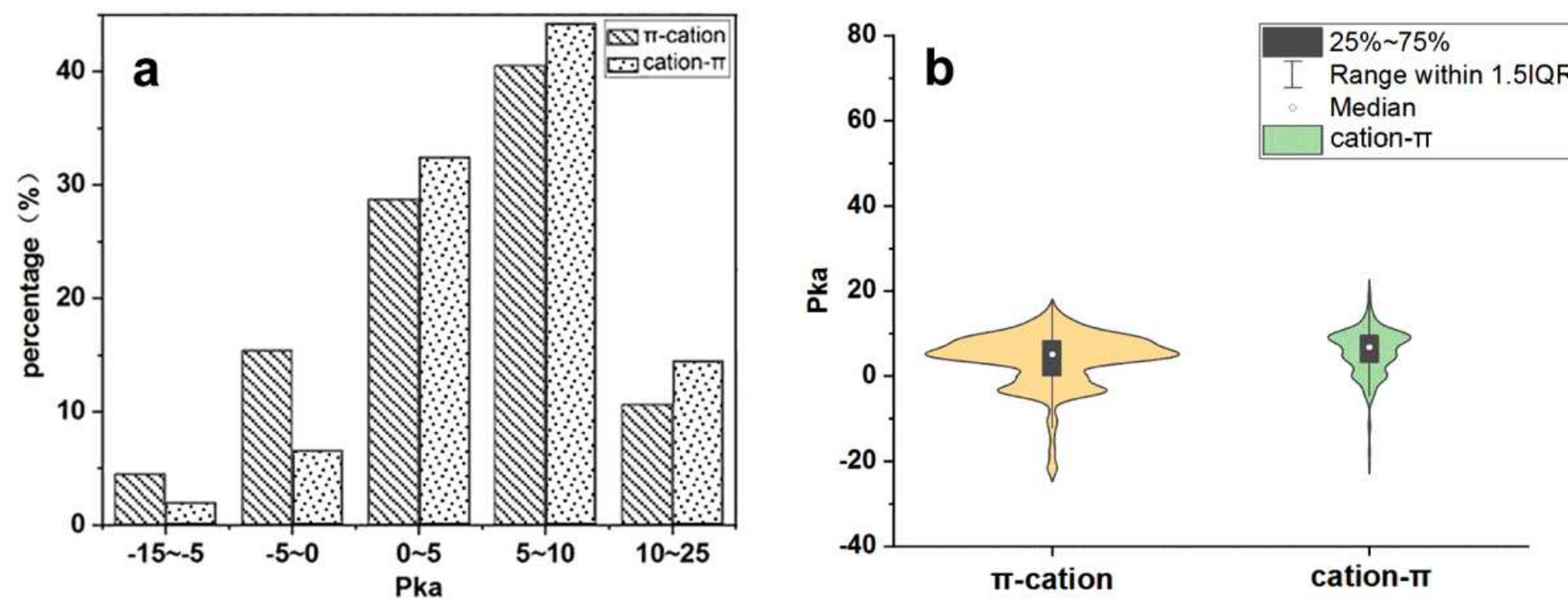

Figure 4

The histogram (a) and violin plot (b) of ligand pKa in cation- $\pi$ and $\pi$-cation systems. 

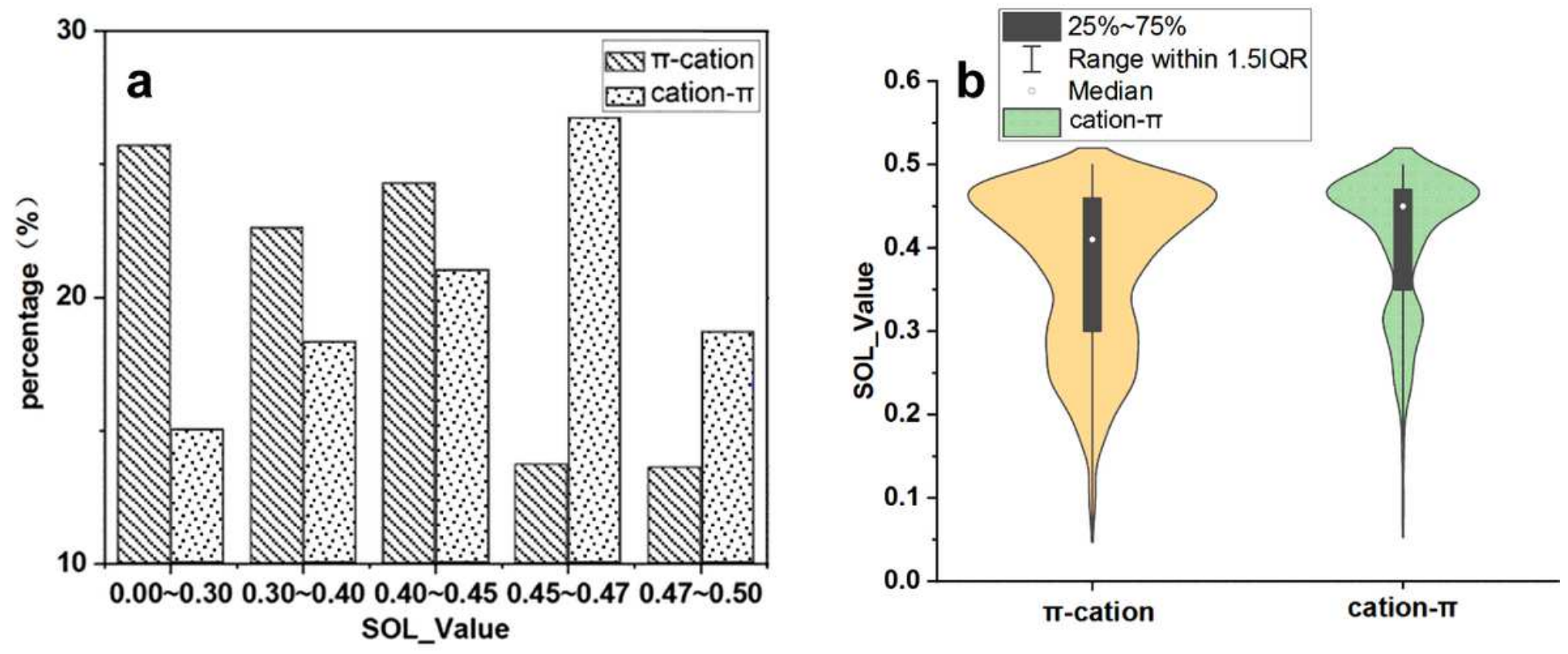

Figure 5

The histogram (a) and violin plot (b) of SOL_Value in cation- $\pi$ and $\pi$-cation systems. 
a

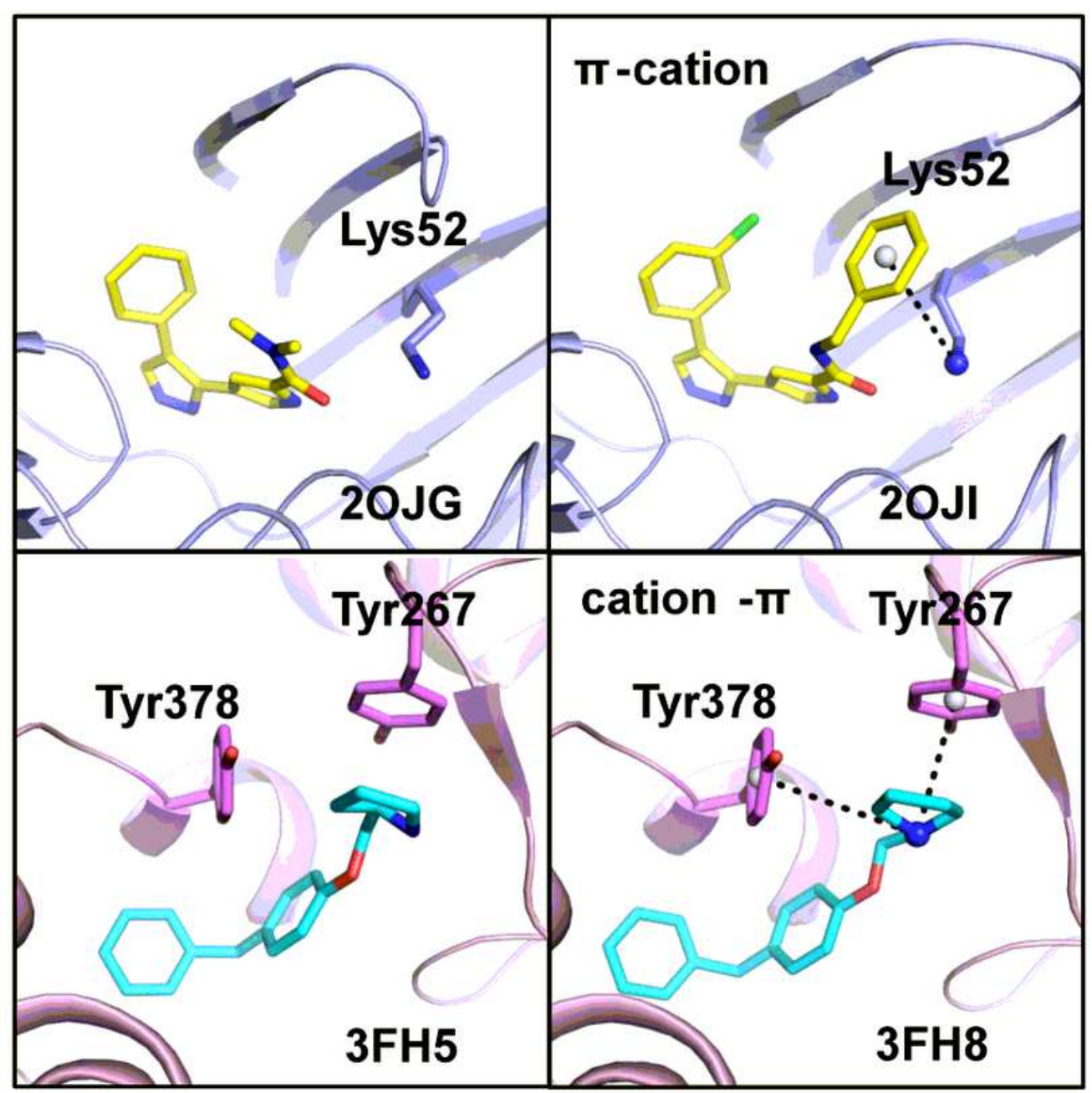

b The comparison of cation $-\pi$ and $\pi$-cation interaction

\begin{tabular}{cccccc}
\hline PDB & $\mathrm{IC}_{50} / K_{i}(\mathrm{nM})$ & Hyd score & SOL_Value & $\pi$ & cation \\
\hline 2OJG & 2300 & 16.39 & 0.15 & & \\
2OJI & 86 & 16.39 & 0.18 & $33 A$ & Lys52 \\
3FH5 & 87 & 42.41 & 0.39 & & \\
3FH8 & 26 & 42.41 & 0.41 & Tyr267/378 & 27P \\
\hline
\end{tabular}

Figure 6

(a) Depicts of cation- $\pi$ and $\pi$-cation interactions and (b) comparison of cation- $\pi$ and $\pi$-cation interactions for IC50/Ki, hydrophobic score (Hyd score), and SOL_Value.

\section{Supplementary Files}

This is a list of supplementary files associated with this preprint. Click to download. 
- supplementarymaterial20200818.doc 